\title{
A Compliance-Based Microflow Stabilizer
}

\author{
Bozhi Yang and Qiao Lin
}

\begin{abstract}
This paper presents a microfluidic device that can passively reduce fluctuations in an upstream fluid flow and generate a largely steady flow in a microfluidic system. The device features a series of compliant membranes that form deformable walls of a microchannel. For an incoming pulsatile flow, passive vibrations of the membranes allow the fluid to be accumulated for an overflow and discharged for an underflow, resulting in drastically reduced fluctuations in flow rate and pressure. A lumped-parameter model is developed to simulate the device characteristics, in which the coupling of membrane vibrations and fluid flow in individual channel sections associated with a single membrane is first represented by squeeze-film theory and inertia-free structural dynamics. The entire device is next represented as a system by connecting individual channel sections in series. The model is numerically solved, and the results agree with experiments. The theoretical and experimental results both show that a five-membrane device can stabilize a pulsating flow by a factor of about 20 . These results also reveal that smaller average flow rate, smaller fluctuation, and higher fluctuation frequency lead to more effective flow stabilization. With these characteristics, the flow stabilizer can be used to obtain steady flow in microfluidic systems.

[2008-0305]
\end{abstract}

Index Terms-Compliant membrane, flow rectification, flow stabilization, fluid-structure interaction, lumped-parameter model.

\section{INTRODUCTION}

$\mathbf{L}$ AB-ON-A-CHIP systems involve integration and automation of many individual steps performed in macroscale biochemical analysis on a small chip that typically contains fluid flow in a fluidic circuit [1]-[3]. A lab-on-a-chip device generally uses a host of fluid manipulation tools that are of different levels of complexity, such as fluid pumping, mixing, valving, gating, and rectification [1]-[3], as well as flow oscillation [4] and fluid interface control [5]. For many labon-chip systems, the ability to precisely transport reagents to different locations at steady rate and controlled dosage is important. To deliver fluid through the fluid circuits, various micropumps have been developed; however, most micropumps are mechanical ones, i.e., check-valved or peristaltic pumps, which typically generate a pulsatile fluid flow. Therefore, it would be interesting to develop flow stabilization devices to rectify the pulsatile flow components generated by these pumps.

Manuscript received December 13, 2008; revised March 26, 2009. Current version published June 3, 2009. This work was supported in part by the National Science Foundation under Grant CBET-0693274. Subject Editor S. Shoji.

B. Yang is with Infinia Corporation, Kennewick, WA 99336 USA (e-mail: yangbozhi@yahoo.com).

Q. Lin is with the Department of Mechanical Engineering, Columbia University, New York, NY 10027 USA (e-mail: qlin@ columbia.edu).

Color versions of one or more of the figures in this paper are available online at http://ieeexplore.ieee.org.

Digital Object Identifier 10.1109/JMEMS.2009.2021826
However, microflow rectification devices that can stabilize flow (and/or pressure) variations have received rather limited attention although they are important for applications where constant flow rate is required, such as controlled drug delivery [6], microreactors [7], and microdialysis [8]. Recently, two passive microfluidic devices have been reported that can rectify fluctuating fluid flow or pressure [9], [10]. The first device uses a series of hydrophobic capillaries along a microchannel to accumulate liquid during driving pressure oscillations and works as a "pressure source" [9]. Although not specifically designed for flow/pressure rectification, the device can potentially be used for the purpose. This device works only with aqueous solutions and suffers from losing hydrophobicity after a short time. The second device uses compressible air cushion, trapped in a side branch of main channel, to dampen pressure/flow pulse in liquid stream [10]. In both devices, the air/liquid direct contact can cause contamination, air dissolution, and fluid evaporation, leading to device malfunction. In addition, the two devices have relatively low-frequency response because of to the back-and-forth fluid movement inside the narrow capillaries or side channels.

This paper presents a novel passive flow stabilization device that consists of a series of compliant membranes forming walls of a microchannel. As a pulsatile fluid flows through the channel, it induces passive vibrations of the compliant membranes. Such vibrations allow the fluid to be stored by an outward membrane deflection to reduce an overflow, and discharged by an inward deflection to compensate for an underflow. Thus, the fluctuation of fluid flow emerging from the device is drastically stabilized. A lumped-parameter model was developed to predict the device flow stabilization performance. The model considers not only the interactions of membrane vibrations and surrounding fluid flow for individual membranes but also the coupling of such fluid-structure interaction associated with multiple membranes at system level. The model predicts a characteristic frequency of the device, i.e., the minimum frequency for the device to function efficiently. It also simulated the impact of device geometry, fluctuation frequency, average flow rate, and fluctuation amplitude, on the device stabilization performance. By proper design of membrane vibration characteristics, flow stabilization can be achieved over a wide range of fluctuation frequencies, including those at which micropumps commonly operate. The microflow stabilizer is robust due to a simple geometry and passive operation. The compliant membranes eliminate the risk of contamination, fluid evaporation, or air dissolution into fluid. In addition, the device principle is applicable to both liquid and gas, and can be used to remove flow fluctuations in conventional large-scale fluidic systems also.

This paper is organized as follows. The design and fabrication of the microflow stabilizer will first be described in 


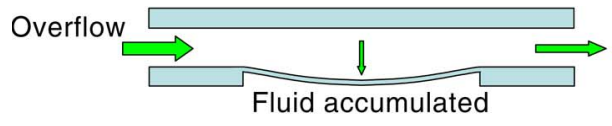

(a)

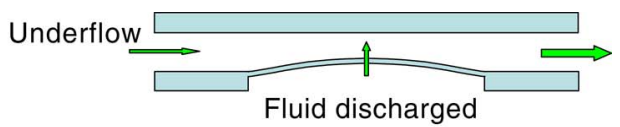

(b)

Fig. 1. Principle of flow stabilization using a one-membrane device as an example. (a) Overflow forces the membrane to move downward, leading to fluid storage. (b) Underflow causes the membrane to move upward, leading to fluid discharge. Thus, a steady flow rate is achieved downstream.

Section II, and the experimental method will be presented in Section III. The stabilizer model and its solution method will be discussed in Section IV. Finally, the modeling and experimental results are presented and compared in Section V.

\section{DESIGN AND FABRICATION}

The microflow stabilizer uses the large compliance of polymer material to achieve passive flow stabilization. The device consists of a microchannel, whose sidewalls are formed by thin compliant membranes. These membranes can be arranged in series or in parallel along the fluid channel. Fig. 1 shows the flow stabilization principle using a one-membrane device, in which a compliant membrane forms the floor of a fluid channel. During operation, an incoming pulsatile flow forces the membrane to vibrate about its equilibrium position at the frequency of flow fluctuations. For an overflow, the hydrodynamic force pushes the membrane to move downwards and some liquid is stored. For an underflow, the membrane moves upwards under membranes' spring force and the stored fluid is discharged. The compliant membrane in the fluid circuit can be thought of as a "fluidic capacitor," analogous to a capacitor in an electrical circuit, for fluid storage and discharge in the face of an unsteady flow. Therefore, the upstream fluctuations in flow rate and pressure can be filtered as the fluid passes through the device. Note that the device works passively with a robust and simple configuration. It is designed with a large ratio of membrane diameter to channel height. Therefore, the squeeze film effect is significant, leading to heavily overdamped membrane vibrations. This ensures that resonance of membrane vibration, which is undesirable for flow stabilization, will not occur.

In this paper, a simple design was chosen that could be easily fabricated to demonstrate the fluid stabilization principle. The design, shown in Fig. 2, consists of three different layers. The top layer is fabricated from polydimethylsyloxane (PDMS) and bears microfluidic features. The bottom layer is a glass cover slip through which holes are drilled to define the compliant diaphragms, which are formed by the middle layer, a thin PDMS sheet.

Fig. 3 shows the fabrication process for the microflow stabilizer, in which three separately fabricated structural layers were bonded together. The top PDMS sheet bearing channel features was fabricated by replica molding [11], [12]. SU-8 2100 (MicroChem, Newton, MA) was patterned on a silicon wafer to serve as the master mold, which was then exposed to trimethylchlorosilane vapor for $1 \mathrm{~min}$ to facilitate the later release of PDMS. Next, a PDMS prepolymer and curing agent (Sylgard 184 Silicone Elastomer Kit, Dow Corning) were mixed in 10:1 weight ratio, degassed and poured onto the mold. After curing, the top PDMS sheet was retrieved, and fluid (a)

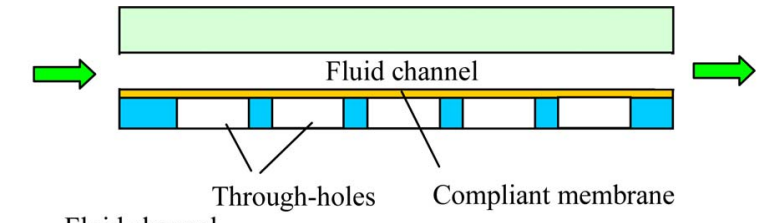

(b)

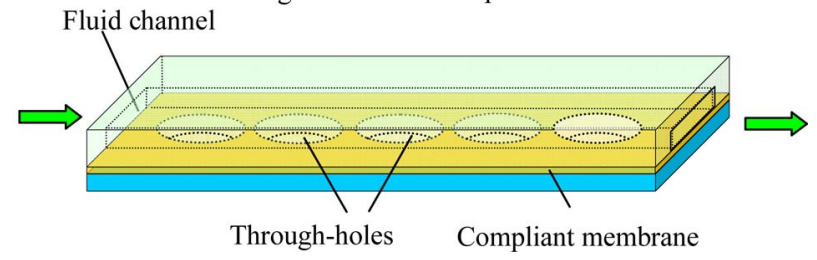

Fig. 2. Schematic of the stabilizer design. (a) Side view and (b) isometric view of a device with five membranes, where a thin PDMS sheet is sandwiched between a PDMS layer bearing a microchannel and a glass cover slip drilled with holes that define the deformable membranes.

(a)

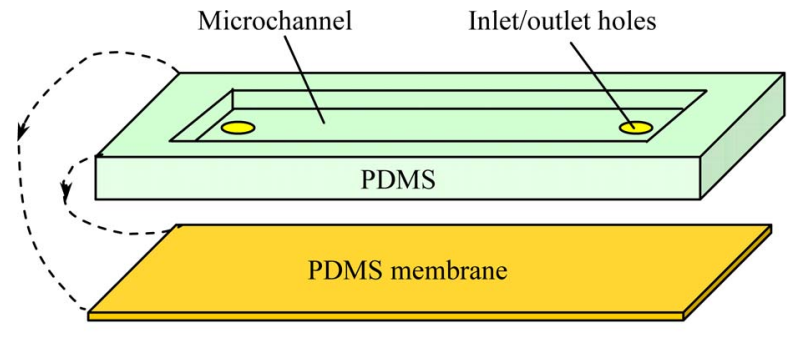

(c)

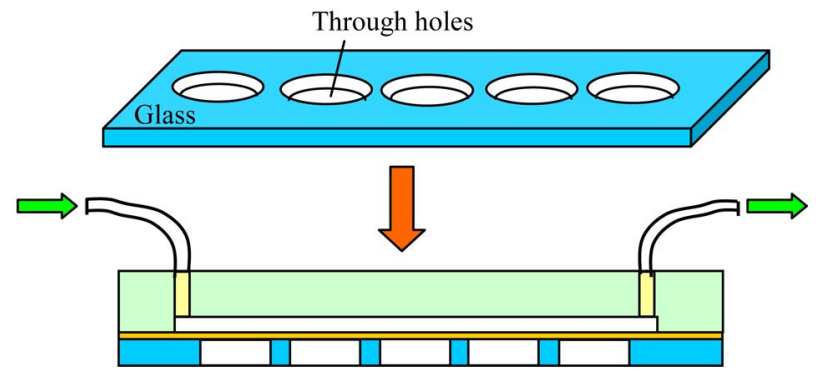

Fig. 3. Fabrication of the prototype device, consisting of: (a) top PDMS sheet containing microchannel features by replica molding; (b) PDMS membrane by spin-coating; (c) holes drilled through the bottom glass plate; and (d) bonding the three layers together and making the micro-to-macro-world interconnections.

interconnection holes were punctured using syringe needles. The thin middle PDMS membrane was fabricated by spin coating and in situ curing PDMS on a silicon wafer. Microscope slide glass was used as the bottom substrate, where throughholes were drilled with diamond-coated drill bits and a regular drill press. The three layers were bonded after oxygen plasma treatment of the bonding surfaces. The bonding sequence is critical to achieving good bonding quality. During the fabrication, the fabricated top PDMS sheet was first bonded onto the thin PDMS membrane, and then the two bonded layers were peeled off together from the underlying silicon wafer using methanol as surfactant. The two bonded PDMS layers were then diced 


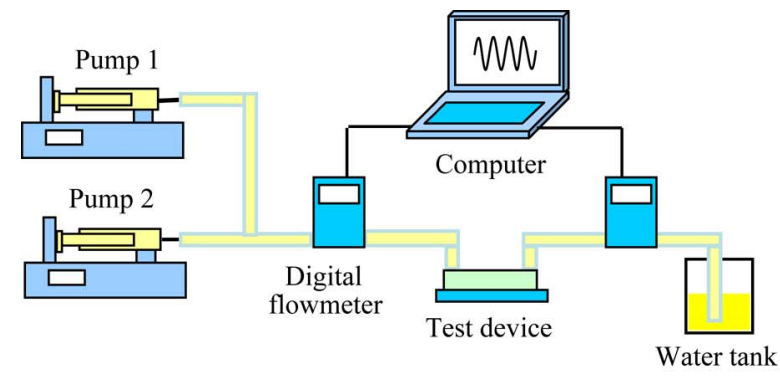

(a)

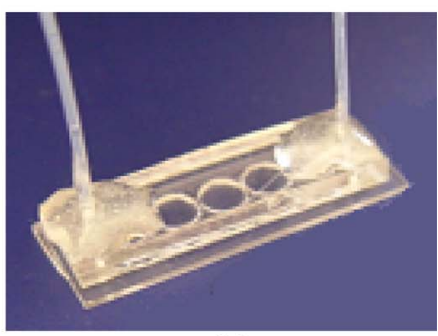

(b)

Fig. 4. (a) Testing setup: two syringe pumps were used to generate the average and fluctuating flow components, respectively, at the device inlet. (b) Photograph of a fabricated prototype device with five circular membranes. (The first and fifth membranes are only partially visible due to the presence of adhesive for the fluidic interconnect tubings.)

and bonded to the bottom glass plate. Fluid interconnections were finally made. Fig. 4 shows images of a fabricated flow stabilizer.

\section{EXPERIMENTS}

Flow stabilization experiments were performed using the setup shown in Fig. 4(a), with a prototype device with five circular membranes [Fig. 4(b)]. Experiments were conducted at room temperature $\left(20^{\circ} \mathrm{C}\right)$ using deionized water. Two syringe pumps (NE-1000, New Era Pump Systems, Inc., Farmingdale, NY and KDS-210, KD Scientific, Inc., Holliston, MA) were, respectively, used to generate the constant and varying components of the inlet flow rate. Therefore, a sinusoidally fluctuating flow with a constant average flow rate was applied at the device inlet, i.e.,

$$
Q_{0}=Q_{\mathrm{avg}}+\Delta Q \cos (2 \pi f t) .
$$

A digital flowmeter (L-1CCM-D, Alicat Scientific, Tucson, AZ was connected to a personal computer on which LabVIEW 7.0 (National Instruments, Austin, TX) is installed to automate flow rate measurements at the device's inlet and outlet. The pressure at the outlet is considered constant at atmosphere pressure, as it was connected to a water tank open to atmosphere. The pressure drop along the Fig. 4(b) device is estimated less than $100 \mathrm{~Pa}$ at the typical flow rate, e.g., $100 \mu \mathrm{L} / \mathrm{min}$. As it is very difficult to supply such a small fluctuating pressure at device inlet, only the device's flow rate stabilization ability was tested, which is characterized by the flow stabilization ratio $R_{\mathrm{f}}$, i.e., ratio of amplitude device inlet and outlet flow rate fluctuation $R_{\mathrm{f}}=\Delta Q_{\text {in }} / \Delta Q_{\text {out }}$.

The KDS-210 syringe pump was used to generate the timevarying inlet flow rate component via reciprocating plunger movement. While forward flow rate fluctuations could be fairly easily generated by forward plunger movement (i.e., pushing), it was more difficult to produce reverse flow rate fluctuations of comparable amplitudes by backward plunger movement (i.e., withdrawal). This is because the plunger withdrawal would lead to a low-pressure region in the syringe, making small air pockets existing in the fluid to drastically expand. In addition, the negative gauge pressure during withdrawal was limited to $1 \mathrm{~atm}$. These difficulties were overcome as follows in the experiment. First, degassed water was used as the testing fluid (a)

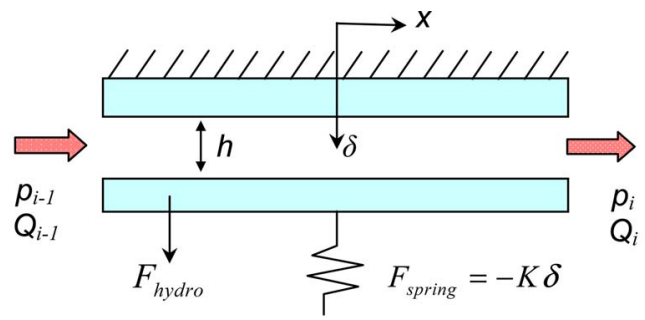

(b)

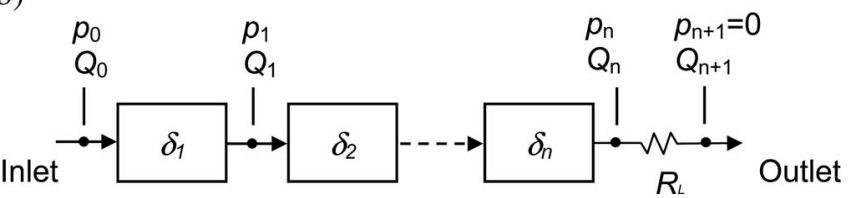

Fig. 5. (a) Model for an individual channel section approximated as a thin fluid film between a stationary rigid plate and a rigid plate attached to an elastic spring with displacement $\delta$. (b) Model is used to represent each channel section of an $n$-membrane microfluidic stabilizer. These channel section representations are concatenated to form a model for the entire device. $R_{\mathrm{L}}$ is the flow resistance of a microfluidic load.

to eliminate entrapped air bubbles in the syringe and tubing. Second, the syringe barrel flange was securely clamped so it could move in both forward and reverse directions at amplitudes defined by the motor. Third, stiff fluid tubings were used to minimize volume expansion. The testing setup thus allowed generation of fluctuating flow rates with amplitudes up to $25 \mu \mathrm{L} / \mathrm{min}$ at frequencies up to $4 \mathrm{~Hz}$.

\section{LumPed-PARAMETER MOdel AND SOLUtions}

A lumped-parameter model was developed to understand the device dynamic characteristics. The model considers the dynamic interaction between vibration of individual membrane and surrounding fluid flow, as well as coupling of such interaction associated with multiple membranes. Coupling of membrane vibration and fluid flow in each individual membrane section is first represented by squeeze-film theory and inertiafree dynamics [13]. The entire device is then represented as a system, in which individual channel sections are connected in series and interact with each other [14]. Fig. 5 shows the model for a $n$-membrane device. In Fig. 5(a), individual channel section associated to a single membrane is modeled as a thin fluid film (thickness $h$ ) between a channel ceiling (represented by a stationary rigid plate) and a flexible membrane (represented by 
a rigid plate attached to an elastic spring with displacement $\delta$ from equilibrium position).

If the membrane mass $m$ is small and vibration frequency is much smaller than natural frequency $f_{\mathrm{n}}$, the membrane inertia is negligible. As damping on the air side is also negligible, the spring-attached plate undergoes quasi-static vibration governed by the following algebraic equation:

$$
K \delta=F_{\mathrm{h}} .
$$

To obtain a closed-form expression of the hydrodynamic force $F_{\mathrm{h}}$, the fluid film is approximated as a square plate with the membrane area $A$. The pressure $p$ is assumed uniform across the film thickness. At low-vibration frequencies, the fluid inertia is negligible, which is justified by a small reduced frequency of the device $\omega \rho h^{2} / \mu$, where $\omega$ is the angular vibration frequency, $h$ the channel height, and $\mu$ the fluid viscosity [15].

The 1-D Reynolds equation then adequately describes the hydrodynamics of the liquid film [16]. In the coordinate frame shown in Fig. 5(a), this equation takes the form

$$
\frac{\partial}{\partial x}\left((h+\delta)^{3} \frac{\partial p}{\partial x}\right)=12 \mu \dot{\delta}
$$

subject to boundary conditions $\left.p\right|_{x=0}=p_{i-1}$ and $\left.p\right|_{x=L}=p_{i}$, $L$ is the effective channel section length, $p_{i-1}$ and $p_{i}$ the pressures upstream and downstream of the $i$ th channel section.

The fluid pressure along the membrane can be calculated from (3) under boundary conditions. Assuming membrane length $L=\sqrt{A}$, the hydrodynamic force is obtained by integration of pressure

$$
F_{\mathrm{h}}=-\mu A^{2} \dot{\delta}_{i} /\left(h+\delta_{i}\right)^{3}+\left(p_{i-1}+p_{i}\right) A / 2 .
$$

Substituting this expression into (3) yields

$$
K \delta_{i}+\mu A^{2} \dot{\delta}_{i}\left(h+\delta_{i}\right)^{3}=\left(p_{i-1}+p_{i}\right) A / 2 .
$$

The flow rate at the channel section inlet and outlet $Q_{i-1}$ and $Q_{i}$ can be calculated from pressure gradient assuming Couette channel flow [16], and the following two relations can be obtained:

$$
\begin{aligned}
& Q_{i-1}+Q_{i}=\left(h+\delta_{i}\right)^{3}\left(p_{i-1}-p_{i}\right) / 6 \mu \\
& Q_{i-1}-Q_{i}=A \dot{\delta}_{i}
\end{aligned}
$$

Equation (7) is also justified as the continuity equation. Therefore, (5)-(7) describe the fluid flow and membrane vibration for the $i$ th channel section.

In an actual fluid circuit, a flow resistor $R_{\mathrm{L}}$, i.e., the total load resistance of the downstream fluid circuit, is usually connected at the device outlet, as shown in Fig. 5(b). Equations (8) and (9) define the model for such flow resistance $R_{\mathrm{L}}$

$$
\begin{gathered}
Q_{n}=Q_{n+1} \\
p_{n}-p_{n+1}=R_{\mathrm{L}} Q_{n+1}
\end{gathered}
$$

where $R_{\mathrm{L}}=\Delta p / Q=8 \mu l_{\mathrm{t}} /\left(\pi r^{4}\right)$ for a tubing of length $l_{\mathrm{t}}$ and inner diameter $r$ [16].
Concatenating the $n$ individual channel sections and the outlet flow resistor $R_{\mathrm{L}}$, a system-level model is obtained for an $n$-membrane device connected to an outlet load resistor [Fig. 5(b)]. The lumped-parameter model is described by a system of ordinary differential equations (ODEs) that can be numerically solved given suitable inlet/outlet boundary conditions. Note that the system-level model in Fig. 5(b) does not include an inlet flow resistance, because the flow rate applied at the device inlet is known in the model. Including an inlet flow resistance will only lead to a known pressure drop across it without changing the device's stabilization performance.

To numerically solve (5)-(7), define $\psi_{i-1}$ and $\psi_{i}$ as the volume flux in and out of the $i$ th channel section from the starting moment of simulation. The membrane deflection and flow rate can then be written as

$$
\delta_{i}=\left(\psi_{i-1}-\psi_{i}\right) / A \text { and } Q_{i}=\dot{\psi}_{i}
$$

Therefore, (5) and (6) take the form of the following equations:

$$
\begin{gathered}
K \cdot \frac{\left(\psi_{i-1}-\psi_{i}\right)}{A^{2}}+\frac{\mu}{\left(h+\delta_{i}\right)^{3}}\left(\dot{\psi}_{i-1}-\dot{\psi}_{i}\right) \\
=\frac{1}{2}\left(p_{i-1}+p_{i}\right) \\
\frac{6 \mu}{\left(h+\delta_{i}\right)^{3}}\left(\dot{\psi}_{i-1}+\dot{\psi}_{i}\right) \\
=p_{i-1}-p_{i} .
\end{gathered}
$$

Given inlet flow rate $Q_{0}$ and outlet pressure $p_{n+1}$, the ODE system can be numerically solved by MATLAB for volume flux $\psi_{i}$, membrane vibration $\delta_{i}$, fluid flow $Q_{i}$ and pressure $p_{i}$, at any location within the device. The numerical results can be used to study the impact of geometry (i.e., membrane diameter, thickness and channel height, etc.) and material properties (i.e., membrane stiffness, fluid viscosity, etc.) on flow stabilization characteristics. For example, they can be used to calculate the flow and vibration characteristics at any locations of the device, and can predict the impact of the inlet flow rate (i.e., average flow rate, fluctuation amplitude, and frequency) and outlet flow resistance on device performance.

Assuming small membrane deflection, i.e., $\delta<<h$, (5) can be linearized and a characteristic frequency $f_{\mathrm{c}}=\omega_{0} / 2 \pi=$ $K h^{3} / 2 \pi \mu A^{2}$ can be defined, which represents the minimum fluctuation frequency for the stabilizer to function efficiently. Flow and pressure stabilization occurs when the fluctuation frequency $(f)$ is much larger than the characteristic frequency, i.e., membrane vibrations are dominated by viscous damping. The characteristic frequency is determined by the device dimension, membrane stiffness, and viscosity of the fluid. To achieve a small characteristic frequency so that the device is applicable to relatively low-frequency flow fluctuations, it is desired that the device has a small channel height and small membrane stiffness as attainable by thinner, larger, and softer membranes.

\section{RESUlts AND Discussion}

In this section, the modeling and testing results will be presented for the five-membrane device shown in Fig. 4(b) with the 
following dimensions: membrane radius $R=2.5 \mathrm{~mm}$, thickness $h_{\mathrm{m}}=48 \mu \mathrm{m}$, edge-to-edge separation $s=0.5 \mathrm{~mm}$, microchannel height $h=110 \mu \mathrm{m}$ high, width $w=5 \mathrm{~mm}$ wide, and length $l_{\mathrm{ch}}=30 \mathrm{~mm}$. The outlet tubing has an inner radius $r$ of $0.4 \mathrm{~mm}$ and length $l_{\mathrm{t}}$ of $50 \mathrm{~mm}$. Thus, the device itself has a nominal flow resistance of $R_{\mathrm{ch}}=12 \mu l_{\mathrm{ch}} /\left(w \cdot h^{3}\right)=$ $4.96 \times 10^{10} \mathrm{~Pa} /\left(\mathrm{m}^{3} / \mathrm{s}\right)$ (assuming no membranes deformation) and the outlet tubing corresponds to an outlet flow resistance $R_{\mathrm{L}}$ of $8 \mu l_{\mathrm{t}} /\left(\pi r^{4}\right)=4.98 \times 10^{9} \mathrm{~Pa} /\left(\mathrm{m}^{3} / \mathrm{s}\right)$. The pressure drop $\Delta p$ along the device and the outlet tubing was estimated about 80 and $8 \mathrm{~Pa}$, respectively, at a typical flow rate of $100 \mu \mathrm{L} / \mathrm{min}$. Other parameters in the simulation include the following: Young's modulus of PDMS $E=750 \mathrm{kPa}$, Poisson's ratio $\nu=0.49$, density $\rho_{\text {PDMS }}=920 \mathrm{~kg} / \mathrm{m}^{3}$; viscosity of water $\mu=0.001 \mathrm{~Pa} \cdot \mathrm{s}$, density $\rho_{\mathrm{w}}=1000 \mathrm{~kg} / \mathrm{m}^{3}$. The characteristic frequency of the device $f_{\mathrm{c}}=K h^{3} / 2 \pi \mu A^{2}$ was estimated $\sim 0.01 \mathrm{~Hz}$.

As the in-plane stress of the PDMS membranes is negligible due to its large compliance, the membrane deflection can be approximated assuming a uniformly loaded circular plate with large deflections [17], i.e., $\delta=0.662 R \sqrt[3]{q R / E h_{\mathrm{m}}}$. This corresponds to a nonlinear spring coefficient $K(\delta)=$ $A h_{\mathrm{m}} \delta^{2} E /\left(0.662^{3} R^{4}\right)$ to be used in (11). Assuming a clamped circular membrane, the undamped natural frequency of the membrane $\left(f_{\mathrm{n}}=10.2158 \sqrt{D /\left(\rho h_{\mathrm{m}}\right)} / R^{2}\right)$ is about $120 \mathrm{~Hz}$ [18] where $D$ is the flexural rigidity of the membrane. Since the fluctuation frequency $f(<5 \mathrm{~Hz})$ is much lower than $f_{\mathrm{n}}$ and typical operating angular frequency $\omega \sim 30$ is much lower than $\mu /\left(\rho h^{2}\right) \sim 100$, the previous assumptions that both the membrane and fluid inertia can be ignored are justified.

In the following, simulation result of the Fig. 4(b) device under the fluctuating inlet flow condition (i.e., fluctuating inlet flow rate $Q_{0}=Q_{\mathrm{avg}}+\Delta Q \cos (2 \pi f t)$ and outlet pressure $p_{6}=0$ ) will be first presented. These include prediction of flow rates, interface pressures, and membrane deflection at different device locations. Then, the predicted outlet flow rate at device outlet will be compared with measurements. Finally, the effect of average flow rate $Q_{\text {avg }}$, flow rate fluctuation amplitude $\Delta Q$ and fluctuation frequency $f$, on the flow stabilization ratio $R_{\mathrm{f}}$. were simulated and compared with testing results.

Fig. 6 shows the calculated time courses of flow rates, interface pressures, and membrane deflection at different positions within the five-membrane device under the following simulation conditions: average flow rate $Q_{\text {avg }}=50 \mu \mathrm{L} / \mathrm{min}$; flow rate fluctuation amplitude $\Delta Q=20 \mu \mathrm{L} / \mathrm{min}$; fluctuation frequency $f=2.5 \mathrm{~Hz}$. The numerical simulation results predict that the flow stabilizer has good capability to stabilize a pulsatile flow rate. As shown in Fig. 6, the computation result reaches steady state within about $5 \mathrm{~s}$. After reaching steady state, the fluctuation fluctuations in flow rate, pressure, and membrane deflection generally decrease along the flow. This yields a flow rate stabilization ratio $R_{\mathrm{f}}$ of about 16.4 , which is in good agreement with the measured value of 14.5 (Fig. 7). The numerically computed pressure stabilization ratio $R_{\mathrm{p}}$ is about 4.4 .

A five-membrane prototype device [Fig. 4(b)] which has the same dimension as that simulated in Fig. 6 was tested. The influence of inlet flow rate conditions, i.e., the average flow rate

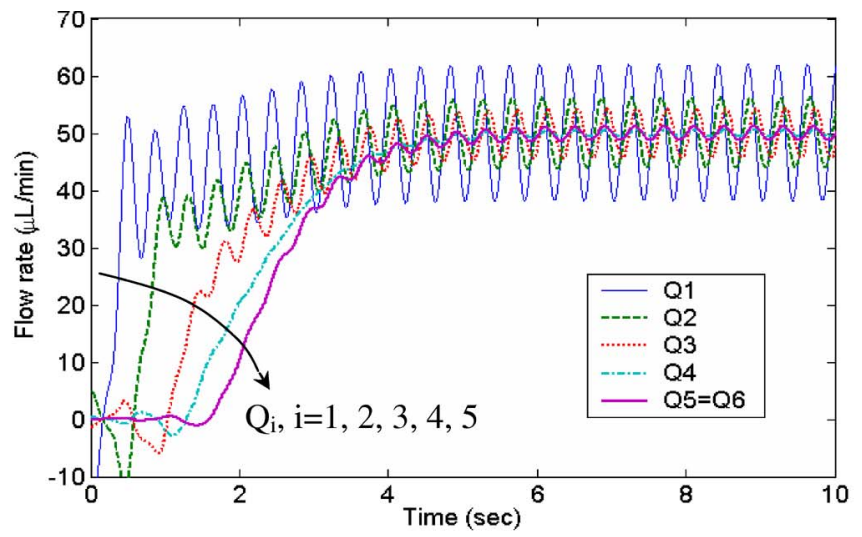

(a)

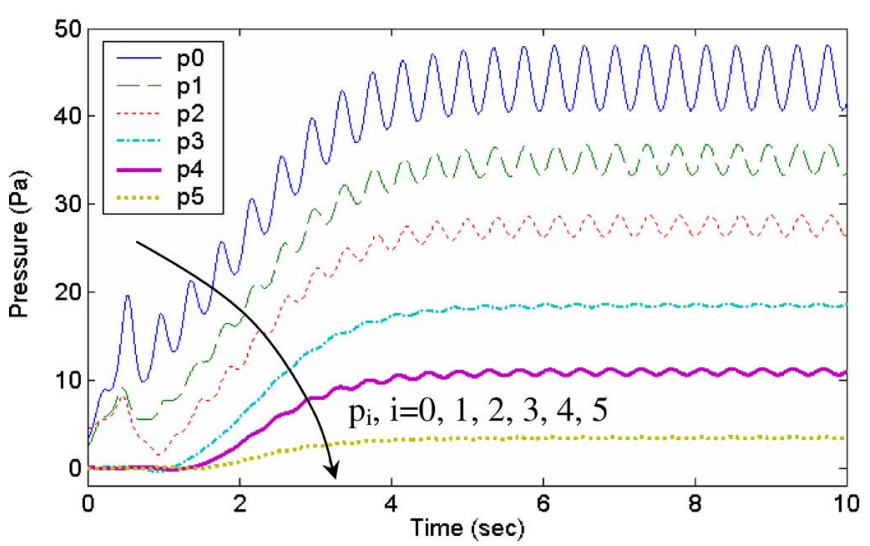

(b)

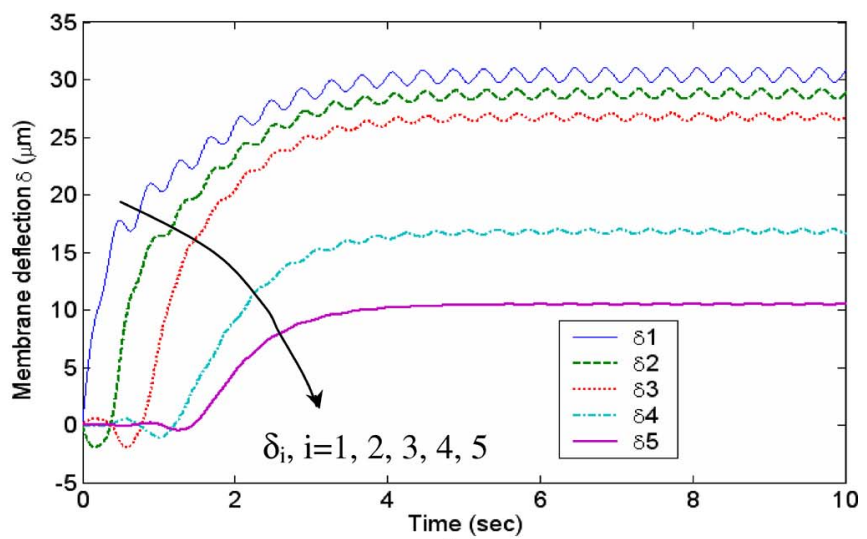

(c)

Fig. 6. Simulated time courses of (a) flow rates, (b) interface pressures, and (c) membrane deflection at different positions within the Fig. 4(b) device (simulation condition: fluctuating inlet flow rate $Q_{0}=Q_{\text {avg }}+\Delta Q \cos (2 \pi f t)$, where $Q_{\mathrm{avg}}=50 \mu \mathrm{L} / \mathrm{min} ; \Delta Q=20 \mu \mathrm{L} / \mathrm{min} ; f=2.5 \mathrm{~Hz}$, and outlet pressure $p_{6}=0$ ).

$Q_{\mathrm{avg}}$, the flow rate fluctuation amplitude $\Delta Q$ and fluctuation frequency $f$, on the flow stabilization ratio $R_{\mathrm{f}}$, were studied. These factors are significant particularly because of the spring stiffening effects of the compliant membranes. To facilitate comparison, the experimental data and numerical results will be presented in the same figures.

To demonstrate the device's flow stabilization capability, the device was first tested under the same inlet flow condition as used in the numerical simulation of Fig. 6: $Q_{\mathrm{avg}}=50 \mu \mathrm{L} / \mathrm{min}$; $\Delta Q=20 \mu \mathrm{L} / \mathrm{min} ;$ and $f=2.5 \mathrm{~Hz}$. Fig. 7 shows the measured 


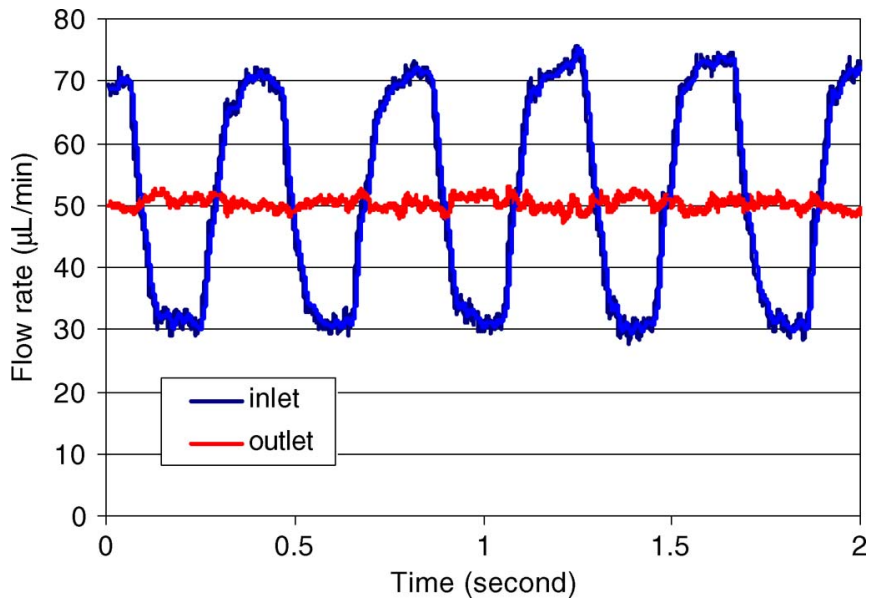

Fig. 7. Measured time course of inlet and outlet flow rates for the fivemembrane device in Fig. 4(b) $\left(Q_{\text {avg }}=50 \mu \mathrm{L} / \mathrm{min} ; \Delta Q=20 \mu \mathrm{L} / \mathrm{min} ; f=\right.$ $2.5 \mathrm{~Hz}$; flow rate stabilization ratio $R_{\mathrm{f}} \approx 14.5$ ).

time course of the inlet and outlet flow rates of the device during operation. The resulting flow rate stabilization ratio was calculated to be $R_{\mathrm{f}} \approx 14.5$, which is close to the numerical calculated value of 16.4 shown in Fig. 6. As shown in Fig. 7, the fluctuation amplitude at the outlet was drastically reduced from 20 to about $1.4 \mu \mathrm{L} / \mathrm{min}$. Relative to the average flow rate $Q_{\text {avg }}$, flow rate fluctuations $\Delta Q$ were reduced from $40 \%$ at the inlet to about $2.5 \%$ at the outlet. This demonstrates that the device is indeed capable of stabilizing fluctuating flow, and the tested stabilization ratio is in good agreement with the numerical calculated value.

The flow rate stabilization ability of the device was further studied by varying the device's inlet average flow rate $Q_{\text {avg }}$, inlet fluctuation amplitude $\Delta Q$, and fluctuation frequency $f$. The inlet flow rate condition influences the dynamic characteristics of the membrane vibrations, and will thus have strong impact on the device's flow stabilization ratio $R_{\mathrm{f}}$. Fig. 10 shows the experimental data and numerical results of the influence of average flow rate $Q_{\text {avg }}$ on flow stabilization ratio $R_{\mathrm{f}}$. The test and modeling conditions used were the same for the inlet flow rate: fluctuation amplitude $\Delta Q=40 \sim 50 \mu \mathrm{L} / \mathrm{min}$ and fluctuation frequency $f=2.5 \mathrm{~Hz}$. Experiments and modeling results are in good agreement in that the flow stabilization ratio $R_{\mathrm{f}}$ increases with average flow rate $Q_{\text {avg decreasing. As shown }}$ in Fig. 8, the tested flow stabilization ratio $R_{\mathrm{f}}$ increases from 3.1 to 6.9 as the average flow rate $Q_{\text {avg }}$ decreases from 434 to about $53 \mu \mathrm{L} / \mathrm{min}$. Similarly, the numerically simulated flow stabilization ratio $R_{\mathrm{f}}$ increases from 2.8 to 8.6 as the average flow rate $Q_{\text {avg }}$ decreases from 450 to about $50 \mu \mathrm{L} / \mathrm{min}$.

The continuous drop of flow stabilization ratio $R_{\mathrm{f}}$ with increasing average flow rate $Q_{\text {avg }}$ is due to the nonlinear behavior of the membrane vibrations. An incoming pulsatile flow rate forces the membrane to vibrate around a static equilibrium position that is determined by the average flow rate $Q_{\text {avg. At }}$ larger $Q_{\text {avg }}$, this equilibrium position moves outward more and the membrane become much stiffer due to large deformation effects. Thus, membrane vibration amplitudes will be reduced, resulting in a decrease in stabilization ratio. In numerical simulation, this effect was accounted for by using a nonlinear spring

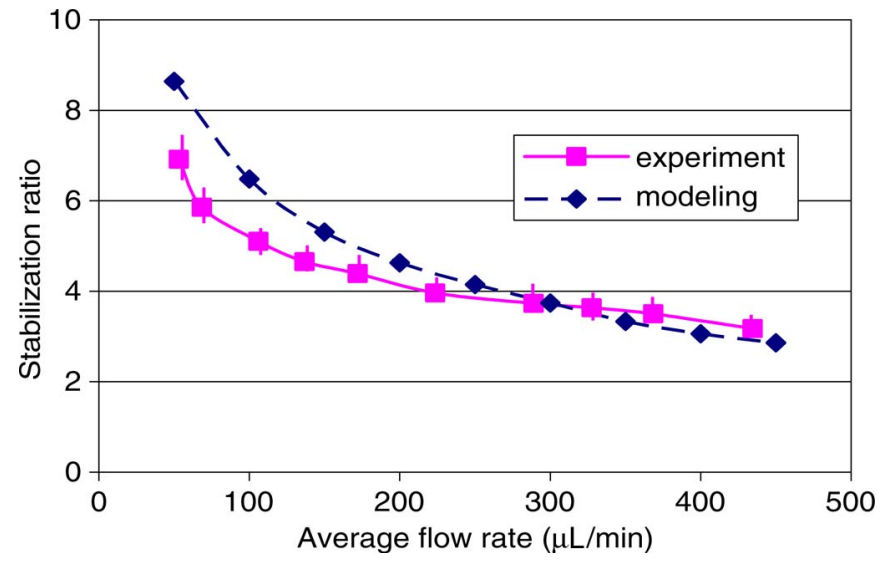

Fig. 8. Influence of average flow rate on the stabilization ratio (test condition: $\Delta Q=40 \sim 50 \mu \mathrm{L} / \mathrm{min} ; f=2.5 \mathrm{~Hz}$ ).

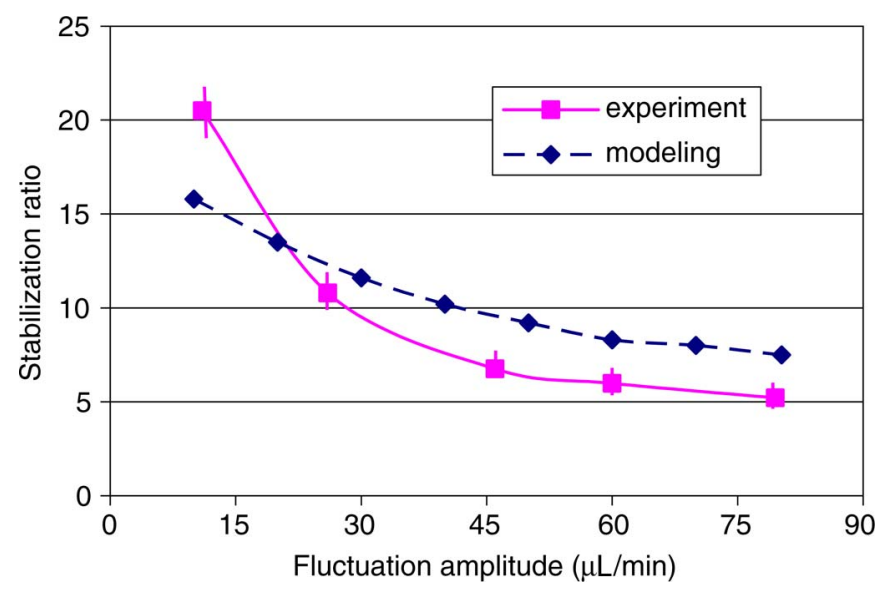

Fig. 9. Influence of flow rate fluctuation on flow stabilization ratio (test condition: $Q_{\text {avg }}=90 \mu \mathrm{L} / \mathrm{min} ; f=2.5 \mathrm{~Hz}$ ).

coefficient $K(\delta)$ of the membrane deflection. Thus, the model accurately considers the membrane vibration physics.

Fig. 9 shows the experimental and numerical results of the influence of the inlet flow fluctuation amplitude $\Delta Q$ on the flow stabilization ratio $R_{\mathrm{f}}$. The test conditions and the modeling parameters were the same for the inlet flow rate: average flow rate $Q_{\text {avg }}=90 \mu \mathrm{L} / \mathrm{min}$ and fluctuation frequency $f=2.5 \mathrm{~Hz}$. Experimental data demonstrate that the flow stabilization ratio $R_{\mathrm{f}}$ increases from 5.2 to 20.5 as the fluctuation amplitude $\Delta Q$ decreases from 79.5 to $11 \mu \mathrm{L} / \mathrm{min}$. Numerical results show a similar trend as the experiment data, where the flow stabilization ratio $R_{\mathrm{f}}$ increases from 7.5 to 15.8 as the fluctuation amplitude $\Delta Q$ decreases from 80 to $10 \mu \mathrm{L} / \mathrm{min}$.

It is believed that decreasing of flow stabilization ratio $R_{\mathrm{f}}$ with increasing fluctuation amplitude $\Delta Q$ is also caused by the large membrane deformation effect. As $\Delta Q$ increases, the membrane vibration amplitude will increase consequently, but the increase in membrane vibration amplitude cannot follow the increase in $\Delta Q$ because the membranes become stiffer. This phenomenon can also be attributed to the spring stiffening effect of the membrane. Overall, increasing value of inlet fluctuation amplitude $\Delta Q$ will lead to a reduction in $R_{\mathrm{f}}$.

The frequency response of the flow stabilizer was finally studied. The influence of fluctuation frequency $f$ on the flow 


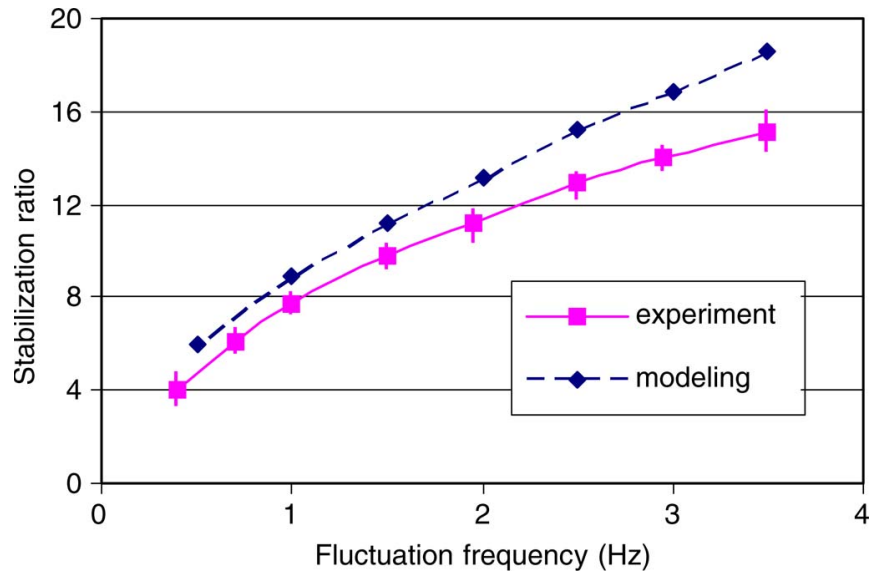

Fig. 10. Influence of fluctuation frequency on the flow stabilization ratio (test condition: $Q_{\mathrm{avg}}=50 \mu \mathrm{L} / \mathrm{min} ; \Delta Q=30 \mu \mathrm{L} / \mathrm{min}$ ).

stabilization ratio $R_{\mathrm{f}}$ is shown in Fig. 10. The testing conditions and simulation parameters were the same for the inlet flow rate: average flow rate $Q_{\mathrm{avg}}=50 \mu \mathrm{L} / \mathrm{min}$ and fluctuation amplitude $\Delta Q=30 \mu \mathrm{L} / \mathrm{min}$. Both experiments and simulation results show that the flow stabilization ratio $R_{\mathrm{f}}$ increases with the fluctuation frequency $f$ for the specific frequency range. It can be seen that the tested flow stabilization ratio $R_{\mathrm{f}}$ increases from 4 to 15.1 as the fluctuation frequency $f$ increases from 0.4 to $3.5 \mathrm{~Hz}$. Similarly, the simulated flow stabilization ratio $R_{\mathrm{f}}$ increases from 6 to 18.6 as the fluctuation frequency $f$ increases from 0.5 to $3.5 \mathrm{~Hz}$.

It is believed that the device has an optimal operating frequency $f_{\text {opt }}$, corresponding to the maximum $R_{\mathrm{f}}$ under given $Q_{\text {avg }}$ and $\Delta Q$. The device has low flow stabilization ratio at frequencies close to characteristic frequency $f_{\mathrm{c}}$, at which the membranes do not respond sensitively to flow fluctuations. At very high frequencies, the inertia effects of the membrane and fluid that are neglected in numerical simulation will be significant, causing the decay of the vibration amplitude. Fig. 10 shows a trend that the device will be optimal at a frequency higher than $3 \mathrm{~Hz}$, which were not tested due to instrument limitations.

The measured flow stabilization ratios $R_{\mathrm{f}}$ are generally in good agreement with the numerical results. However, both the experimental and numerical results are much smaller than the ratios by the analytical prediction. This can be attributed to the nonlinear behavior of membrane vibrations that was not considered by the assumptions used in the analytical prediction. The analytical model assumes linear membrane behavior; however, the fact is, as the average flow rate and fluctuation amplitude increase, the membranes vibrations become increasingly nonlinear due to the membrane stiffening effect. Thus, the membrane vibration characteristics are changed, leading to a lower stabilization ratio. Additionally, errors in outlet flow rate measurements, due the presence of the relatively large flowmeter noise (about $\pm 3 \mu \mathrm{L} / \mathrm{min}$; flowmeter reading resolution: $1 \mu \mathrm{L} / \mathrm{min}$ ), may have led to significant inaccuracies in the measured flow stabilization ratios. The actual value is believed to be higher.

From the model, the device geometry can be varied to improve the performance of the device. For example, the flow stabilization ratio of the flow stabilizer can be improved by using thinner and larger membranes, and using channels of smaller height to allow smaller $f_{\mathrm{c}}$. In addition, the compliant membranes can be arranged on both floor and ceiling of the microchannel. These issues and opportunities should be investigated in future work.

\section{CONCLUSION}

A microflow stabilizer has been developed and modeled. The device utilizes compliant membranes whose vibrations are able to remove fluctuations of an incoming pulsatile fluid flow. Experiments have demonstrated that the device can effectively stabilize a pulsating flow with a flow stabilization ratio up to 20 for a five-membrane device. A lumped-parameter model was developed to study the dynamic device characteristics. The model predicts a characteristic frequency of the device, i.e., the minimum frequency for the device to function efficiently. The model can be numerically solved using the experimentally used device dimensions and material properties to account for the nonlinear behavior of the device performance. Both test and simulation results demonstrate that smaller average flow rates, smaller fluctuations, and higher fluctuation frequencies lead to improved flow stabilization. The microflow stabilizer can be used in various lab-on-chip systems that require steady flow rates, such as microreactors, drug delivery, and microdialysis. The device can potentially be used along with most mechanical micropumps. Generally applicable to virtually any fluids, device is passive, highly robust, and has fast time response without risk of cross contamination. Moreover, the stabilization concept can be used to produce designs with much reduced dimensions, since (5)-(7) are based on the squeeze-film model and inertia-free vibration theory, whose validity is, in general, enhanced at reduced length scales so long as continuum-based fluid flow representation is valid.

Devices of other configurations can be designed with the same principle. For example, the compliant membranes can be arranged on more channel walls. To this end, a single-layer replica molding approach could be used to create deformable thin membranes on the vertical sidewalls of microchannel [19], [20]. Generally, the larger the area of channel walls this is formed by compliant membranes (of the same thickness and material properties), the better the flow stabilization performance. The device could even be designed as elastic tubing to mimic the mammalian artery that can stabilize blood flow and pressure pulses generated by heart pulsation due to the elastic deformation of artery tube wall. In addition, the membranes could be arranged in series or in arrays along the fluid channel with different size and thickness. This is to account for the decreasing hydrodynamic pressures along the flow.

There are, however, several issues need to be further addressed. First, the membranes were modeled as square plates in applying the Reynolds equation. To improve the current model, actual geometry should be used in the future work. A second issue is concerned with the testing of the device's pressure stabilization capability, which can be realized by using accurate pressure transducers and/or controller. Third, it will also be interesting to characterize the membrane vibrations, 
which will give more insight to the nonlinear behavior of membrane, and verify the membrane stiffening effects. Finally, while a lumped-parameter model reveals the general trend in the device's performance, it does not reveal the exact physics with each membrane that is simplified as a rigid plate attached to a spring. To better model the device physics, 3-D computational fluid dynamics simulation considering transient structure-fluid interaction is desired. This may be pursued in future work.

\section{ACKNOWLEDGMENT}

The authors would like to thank the MEMS Laboratory, Department of Electrical and Computer Engineering, Carnegie Mellon University, for generously granting access to its fabrication and characterization facilities.

\section{REFERENCES}

[1] N. T. Nguyen and S. T. Wereley, Fundamentals and Applications of Microfluidics, 2nd ed. Boston, MA: Artech House, 2006.

[2] S. Shoji and M. Esashi, "Microflow devices and systems," J. Micromech. Microeng., vol. 4, no. 4, pp. 157-171, Dec. 1994.

[3] G. T. A. Kovacs, Micromachined Transducers Sourcebook. Boston, MA: WCB, 1998.

[4] U. Gebhard, H. Hein, E. Just, and P. Ruther, "Combination of a fluidic micro-oscillator and micro-actuator in LIGA-technique for medical application," in Proc. 12th TRANSDUCERS, Chicago, IL, Jun. 16-19, 1997, pp. 761-764.

[5] B. Kuczenski, P. R. LeDuc, and W. C. Messner, "Pressure driven spatiotemporal control of the laminar fluid interface in a microfluidic network," Lab Chip, vol. 7, no. 5, pp. 647-649, 2007.

[6] B. Wang, T. J. Siahaan, and R. A. Soltero, Drug Delivery: Principles and Applications, 1st ed. Hoboken, NJ: Wiley, 2005.

[7] S. Dewitt, "Microreactors for chemical synthesis," Curr. Opin. Chem. Biol., vol. 3, no. 3, pp. 350-356, Jun. 1999.

[8] R. J. Schutte, S. A. Oshodi, and W. M. Reichert, "In vitro characterization of microdialysis sampling of macromolecules," Anal. Chem., vol. 76, no. 20, pp. 6058-6063, Oct. 2004.

[9] E. D. Hobbs and A. P. Pisano, "Micro capillary-force driven fluidic accumulator/pressure source," in Proc. 12th TRANSDUCERS, Boston, MA, Jun. 8-12, 2003, vol. 1, pp. 155-158.

[10] T. T. Veenstra, N. R. Sharma, F. K. Forster, J. G. E. Gardeniers, M. C. Elwenspoek, and A. van den Berg, "The design of an in-plane compliance structure for microfluidical systems," Sens. Actuators B, Chem., vol. 81, no. 2/3, pp. 377-383, Jan. 2002.

[11] C. Duffy, J. C. McDonald, O. J. A. Schueller, and G. M. Whitesides, "Rapid prototyping of microfluidic systems in poly(dimethylsiloxane)," Anal. Chem., vol. 70, no. 23, pp. 4974-4984, Dec. 1998.
[12] B. H. Jo, L. M. V. Lerberghe, J. N. Motsegood, and D. J. Beebe, "Three-dimensional micro-channel fabrication in poly-dimethylsiloxane (PDMS) elastomer," J. Microelectromech. Syst., vol. 9, no. 1, pp. 76-81, Mar. 2000.

[13] A. Cameron and C. M. Mc Ettles, Basic Lubrication Theory. New York: Halsted, 1981.

[14] Q. Lin, B. Yang, J. Xie, and Y. C. Tai, "Dynamic simulation of a peristaltic micropump considering coupled fluid flow and structural motion," J. Micromech. Microeng., vol. 17, no. 2, pp. 220-228, Feb. 2007.

[15] L. D. Landau and E. M. Lifshitz, Fluid Mechanics, 2nd ed., vol. 6. New York: Pergamon, 1987.

[16] F. M. White, Fluid Mechanics, 5th ed. New York: McGraw-Hill, 2002.

[17] S. P. Timoshenko and S. Woinowsky-Krieger, Theory of Plates and Shells, 2nd ed. New York: McGraw-Hill, 1959.

[18] A. W. Leissa, Vibration of Plates. Washington, DC: GPO, 1969.

[19] N. Sundararajan, D. Kim, and A. A. Berlin, "Microfluidic operations using deformable polymer membranes fabricated by single layer soft lithography," Lab Chip, vol. 5, no. 3, pp. 350-354, Mar. 2005.

[20] B. Yang and Q. Lin, "A planar compliance-based self-adaptive microfluid variable resistor," J. Microelectromech. Syst., vol. 16, no. 2, pp. 411-419, Apr. 2007.

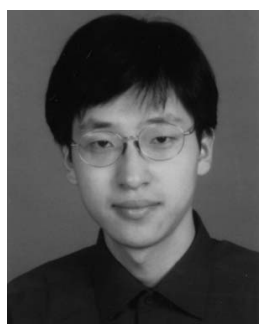

Bozhi Yang received the B.S. degree from Xi'an Jiaotong University, Xi'an, China, in 1997, the M.S. degree from Tsinghua University, Beijing, China, in 2000, and the Ph.D. degree in mechanical engineering from Carnegie Mellon University, Pittsburgh, PA, in 2006, with thesis research on creation and modeling of MEMS fluid control devices.

At Carnegie Mellon, he also developed surfacetension-based microdevices, humidity sensors, and viscosity sensors for glucose monitoring. From 2006 to 2008, he was a Senior Engineer with the Research and Engineering Center, Whirlpool Corporation, Benton Harbor, MI. He is currently an R\&D Engineer with Infinia Corporation, Kennewick, WA, working on the design and modeling of power and heat generation systems and solar thermal engines.

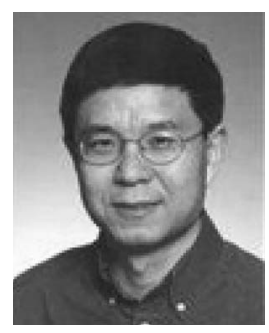

Qiao Lin received the Ph.D. degree in mechanical engineering from California Institute of Technology, Pasadena, in 1998, with thesis research in robotics.

$\mathrm{He}$ conducted postdoctoral research in microelectromechanical systems (MEMS) with the Caltech Micromachining Laboratory from 1998 to 2000, and was an Assistant Professor of mechanical engineering with Carnegie Mellon University from 2000 to 2005. Since 2005, he has been an Associate Professor of mechanical engineering with Columbia University, New York, NY. His research interests are in designing and creating integrated micro/nanosystems, in particular MEMS and microfluidic systems, for biomedical applications. 